\title{
La triple biopsia para carcinoma del seno
}

\author{
Doctor Hugo Quijano M. *
}

Casuística del Instituto Nacional de Cancerología. Bogotá. Comunicación preliminar a la III Convención Nacional de Obstetricia y Ginecología en Ibagué.

La gran incidencia de carcinoma del seno en nuestro medio, segundo lugar después del carcinoma del cervix y tercero después del carcinoma cutáneo y del cuello uterino en las neoplasias en general (1), nos ha guiado a hacer un breve comentario sobre los tratamientos actuales para el carcinoma del seno, y a exponer una línea de conducta para obtener los mejores resultados en el tratamiento curativo del carcinoma del seno.

En un estudio realizado en el Instituto Nacional de Cancerologia (2), se reportan 516 casos durante los años 1946 a 1952, lo cual da un promedio de 73.7 casos por año.

El presente estudio se refiere a 43 casos, relativamente seleccionados, de mayo de 1955 a septiembre de 1957.

La edad de estas pacientes fluctuó de los 27 a los 74 años, siendo más frecuente de los 41 a los 55.

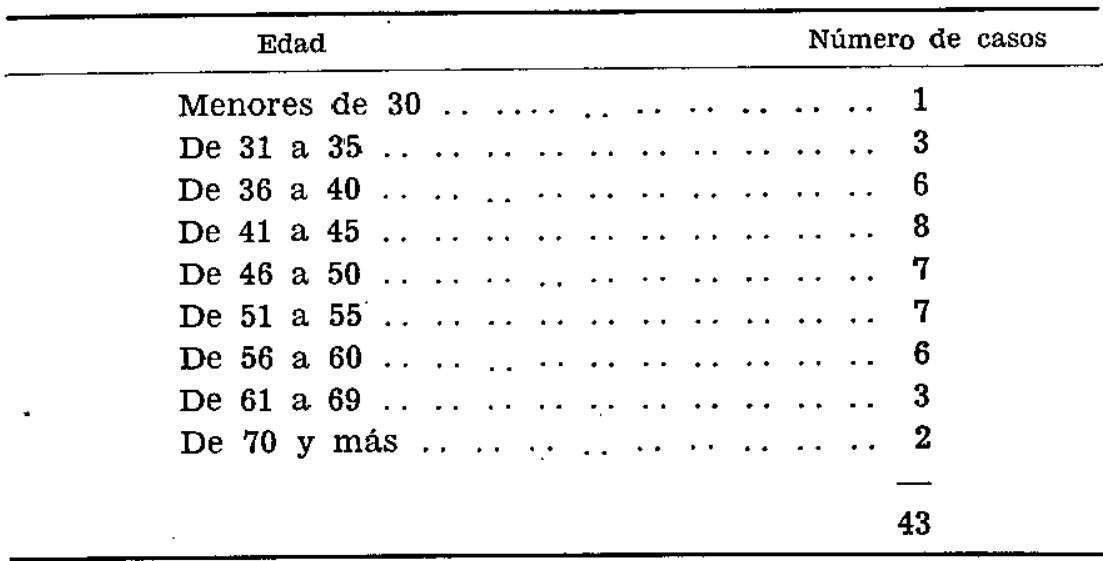

(") Adjunto del Departamento de Ginecología del Hospital de San José, de Bogotá, y del Instituto Nacional de Cancerología. 
De los 43 casos 26 se encontraron localizados en el seno derecho y 17 en el izquierdo. No hubo casọs de localización bilateral.

\begin{tabular}{lllllllllll}
\hline Localización & \multicolumn{1}{c}{} & \multicolumn{4}{c}{ Número de casos } & \multicolumn{1}{c}{ Porcentaje } \\
\hline Derecho & $\ldots$ & $\ldots$ & $\ldots$ & $\ldots$ & $\ldots$ & $\ldots$ & $\ldots$ & 26 & $\ldots$ & 60.4 \\
Izquierdo & $\ldots$ & $\ldots$ & $\ldots$ & $\ldots$ & $\ldots$ & $\ldots$ & & 17 & & 39.6 \\
\hline
\end{tabular}

El tiempo de evolución de esos tumores, hasta cuando las pacientes iniciaron su consulta, varió de los 3 a los 36 meses.

\begin{tabular}{cc}
\hline Evolución en meses & Número de casos \\
\hline 3 & 12 \\
6 & 8 \\
9 & 4 \\
12 & 1 \\
15 & 3 \\
18 & 1 \\
24 & -1 \\
30 & 1 \\
36 & 1 \\
Posterior & -1 \\
$?$ & 43 \\
\hline
\end{tabular}

En realidad este tiempo de evolución es relativo, ya que estas jacientes de un nivel social bajo en su mayoría, y de educación inferior, no dan datos de su evolución que podamos considerar reales, pero dentro de estas cifras podemos darnos cuenta que la gran mayoria de las pacientes no consultaron cuando observaIon sus lesiones. Un dato más desconsolador es que en numerosos casos las pacientes habían consultado su médico, quien por algún motivo había retardado su tratamiento adecuado.

Por este último motivo se me hace importante que este trabajo sea presentado ante esta convención, porque son precisamente el ginecólogo y el obstetra quienes pueden mejor hacer la campaña para el diagnóstico precoz de las lesiones del seno. puesto que es el profesional a quien la paciente deposita su confianza y elimina su pudor natural, que el médico general y aún el cirujano respetan para perjuicio de la misma paciente. 
El tamaño del tumor vàrió de un centímetro a 5 y más, sienco su mayor incidencia entre los 2.5 y 5 centímetros, datos éstos, ce acuerdo también con el tiempo de su evolución y retardö en ia consulta.

\begin{tabular}{|c|c|}
\hline Tamaño del tumor & Número de casos \\
\hline Hasta de 1 centimetro $. . . \therefore: \therefore$ & $-\cdot$ \\
\hline De 1 a 2.5 centímetros $\ldots \ldots \ldots$ & . \\
\hline De 2.5 a 5 centimetros $\ldots \ldots \ldots \ldots$ & 23 \\
\hline Más de 5 centímetros . . . . . . .. & 12 \\
\hline 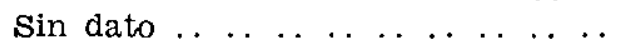 & . \\
\hline
\end{tabular}

Es preciso aclarar en este cuadro que estas pacientes se refieren a un grupo seleccionado como clínicamente operables, de manera que nuestro asombro sería superior al contar aquellas pacientes de mayor evolución y lesiones más avanzadas, que no hemos incluído en este estudio.

Respecto a la localización, se encontraron 15 casos de tumores localizados en el cuadrante superoexterno, 7 en el superointerno, 5 en el inferoexterno, 5 en el inferointerno y 10 de localización central. A uno de los casos, se le había practicado previamente, fuera del Instituto, mastectomía radical.

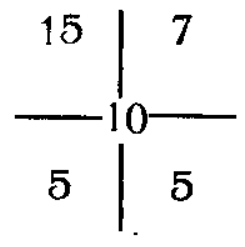

Los tratamientos utilizados hasta la fecha para el tratamiento del cáncer del seno, han sido la cirugia, la radioterapia, el tratamiento sobre glándulas endocrinas (3), ya clínico o quirúrgico, es dẹcir, a base de estrógenos, andrógenos, cortisona, etc., el primero, o de oforectomia, adrenalectomía e hipofisectomia el segundo.

Estos tratamientos se han hecho de acuerdo con el estado clínico, y a este respecto encontramos diversas clasificaciones para catalogar dicho estado, teniendo en cuenta la edad endocrina de la paciente, la naturaleza biológica del tumor, el sitio de invasión y el crecimiento dèl tumor, variando el número de estados o grupos hasta de 8 , inclusive (4). 
Conviene recordar la distribución de los linfáticos del seno, para lo cual haremos un ligero recuento basándonos en parte en un estudio reciente que sobre éstos nọ presentan Tobiassen. Sorensen y Hasner (5).

Los linfáticos del seno se comunican con el plejo linfático de la piel y de la fascia profunda y los troncos linfáticos del seno se drenan por 3 rutas:

1. La ruta principal pasa a los ganglios de la axila a través de troncos laterales que corren a lo largo del borde inferior del pectoral mayor.

2. Una menor parte de los troncos linfáticos pasa por la vía transpectoral a través de los ganglios interpectorales a los gangliọs del vértice de la axila o infraclaviculares.

3. Una mínima parte sigue la ruta a través del músculo pectoral mayor y de los músculos intercostales de los tres primeros espacios a los ganglios paraesternales.

La cadena paraesternal recibe aferentes de los ganglios prepericárdicos situados en la parte anterior de ambos lados del diafragma, de la parte anterior de la pared abdominal y de la cara superior del higado.

Hay anastomosis con la cadena del lado opuesto y con la de los ganglios mediastinales. La distribución de los ganglios es inconstante, pero normalmente se encuentran en los tres primeros espacios.

Los linfáticos de la axila y los paraesternales pasan sepazadamente $o$ anastomosándose a los ganglios supraclaviculares. en la confluencia de las venas yugular interna y subclavia, para llegar "finalmente solos o junto con otros linfáticos del cuello y cel mediastino a la corriente venosa.

Por' este esquema que hemos hecho podemos darnos cuenta con que facilidad; cuando la enfermedad ha alcanzado los ganglics de la mamaria interna, se encuentra prácticamente a un paso de su invasión al mediastino y al hígado, o si ha invadido los ganglios supraclaviculares se encuentra prácticamente generalizada la enfermedad por vía venosa.

Los tratamientos curativos para carcinoma del seno se han limitado a la cirugía y a la radioterapia o su combinación.

Haagensen (6) anota que en los estudios microscópicos sobre los efectos de irradiación para el carcinoma del seno, se requiere minimo una dosis de $4.500 \mathrm{r}$. para producir cambios morfológicos marcạdos, y que aún con las dosis más altas, hasta ahora em- 
pleadas, el carcinoma persiste en aproximadamente cuatro quintas partes de los casos. Las células carcinomatosas se encuentran bloqueadas en un denso estroma fibroso, pero en cualquier momento se pueden hacer libres para crecer de nuevo, lo cual constituye una de las grandes desventajas del tratamiento por irradiación.

Queda, entonces, el tratamiento quirúrgico y el tratamiento combinado.

Las 3 grandes modalidades (7) para el tratamiento del cáncer en la actualidad, son:

1. La mastectomia radical.-Haagensen, en una estadística del Presbyterian Hospital de New York, presenta un porcentaje de curación en los 5 años de $47.9 \%$ y en su estadistica personal de $56.7 \%$ para curación, y de $66.6 \%$ de supervivencia en los 5 años.

2. La mastectomia radical con radioterapia pre y post-operatoria.-Utilizada por Nohrman, del Radiumhemmet en Estocolmo, con un porcentaje de $40.1 \%$.

3. La mastectomía simple con radioterapia post-operatoria. Practicada por Mc Whirter, del Royal Infirmary en Edimburgo, con un porcentaje de $42 \%$.

La mastectomía radical que se practica es más o menos la concebida por Halsted en Baltimore y Willy. Meyer en New York, a fines del siglo pasado, y consiste en extirpar el seno, Ios músculos pectorales y la disección ganglionar de axila.

La técnica de Mc Whirter sería ideal en los casos en los cuales no se encontraron metástasis axilares, pero esto no se puede predeterminar y en caso de que existiesen metástasis axilares. sabemos que es muy incierta la acción de la radioterapia para destruir el carcinoma en los ganglios linfáticos. En el Instituto se le emplea, como veremos al final, únicamente como terapia paliativa en los casos avanzados.

Hay recientemente otros procedimientos quirúrgicos que se podrian llamar mastectomias ultraradicales, tales como la practican Wangensteen (8), en Minneápolis, incluyendo, además de la mastectomía radical, las disecciones supraclavicular y mediastinal, operación practicada en uno o dos tiempos, con una supervivencia en 5 años de un 17\% y Urban en New York, quien practíca la mastectomía radical, resección de la cadena de la mamaria interna y resección costal en un solo bloque, recubriendo el defecto costal con un injerto de fascia lata. Sus resultados, aunciue satisfactorios, tan solo llevan 3 años de observación. 
De lo anterior se deduce que los mejores resultados se han obtenido con la mastectomia radical y las cifras de curación que nos presenta Haagensen son las más altas. En algunas estadísticas es posible que las cifras no sean tan favorables, pero se debe a la técnica aplicada indiscriminadamente, sin la selección de los casos.

Es aquí donde veremos la extraordinaria importancia de Io que se ha descrito como la triple biopsia.

Fue Handley quien en 1922, comenzó a explorar el mediastino, encontrando que en 6 casos había invasión ganglionar en 2 de ellos.

Cinco años más tarde, comentaba que cuando quiera que los ganglios axilares se encontraban invadidos, muy frecuentemente los ganglios de la mamaria interna también lo estaban.

Fue su hijo Richard Handley quien en 1946 (10) comenzó a practicar la disección de la mamaria interna como complemento a la mastectomía radical, presentando en 1954 un estudio muy completo en el cual muestra que en $33 \%$ (sobre 150 casos), presentaba metástasis en la cadena de la mamaria interna.

Si se tenía en cuenta únicamente los casos de metástasis a la axila, encontraba que el $44 \%$ de éstos presentaban metástasis a la mamaria interna, correspondiendo el mayor porcentaje a los casos que presentaban el tumor localizado en la mitad interna del seno.

En una última comunicación (11) el mismo autor, teniendo en cuenta sus estudios anteriores, decía que la mastectomía radical clásica, que tenía en cuenta únicamente el sistema axilar. estaba destinada a fracasar en una tercera parte de los casos, en los cuales no se tenía en cuenta la cadena de la mamaria interna.

Adair (12) sostiene asimismo que se debe practicar la biopsia de la cadena de la mamaria interna en los casos considerados operables, antes de practicar la mastectomía radical.

En 1947. Dahl Iversen, en Copenhague, comenzó a disecar la fosa supraclavicular como complemento al tratamiento quirúrgico del seno. Encontró que había metástasis en ese sitio en un $33 \%$ de los casos. Más tarde, y en pacientes mejor seleccionados, encontró sobre 274 pacientes, metástasis supraclaviculares en un 8.4\%. En todos estos últimos casos, no se apreciaba ninguna metástasis clínicamente.

Basado en todas estas investigaciones, Haagensen, del Presbyterian Hospital de New York, comenzó en 1951 a practicar, como tiempo previo a los casos considerados quirúrgicos, para 
una mayor seguridad en el criterio de operabilidad, lo que él denominó la triple biopsia, es decir, el estudio histopatológico de las biopsias tomadas dei seno, de las cadenas linfáticas de la mamaria interna y de la fosa supraclavicular.

Durante los años 1951 a 1955, en los cuales practicó este método, nos revela los siguientes cuadros:

\begin{tabular}{lcccccc}
\hline & $\begin{array}{c}\text { Región central } \mathrm{y} \\
\text { Sitio biopsia }\end{array}$ & mitad interna & Mitad externa & \multicolumn{2}{c}{ Total } \\
\hline & No & $\%$ & No & $\%$ & No & $\%$ \\
Mamaria I & 63 & 38.9 & 33 & 23.2 & .96 & 31.6 \\
\hline
\end{tabular}

Precisa los factores que favorecen las metástasis de la mamaria interna, en su orden de importancia:

1. Metástasis axilares.

2. Aumento de tamaño del tumor primario.

3. Localización del tumor primario en las regiones central y mitad interna.

\begin{tabular}{|c|c|c|c|}
\hline Sitio biopsia & Número de casos & Número metástasis & Porcentaje \\
\hline Supraclavicular & 110 & 22 & 20 \\
\hline
\end{tabular}

Informa que las metástasis supraclaviculares las encuentra únicamente en las pacientes que presentan metástasis axilares y más tardiamente que la metástasis en la mamaria interna.

Por su experiencia observó, que cuando los genglios del yértice de la axila se encontraban invadidos, el porcentaje de curación por cirugía era mínimo y entonces en 1955 comenzó a practicar la biopsia subclavicular, es decir, del vértice de axila, en vez de la supraclavicular, argumentando que se presentaba una mayor seguridad para determinar la extensión de la vía metastásica a lo lárgo de la ruta axilar supraclavicular.

En el Instituto se comenzó a practicar la triple biopsiz en mayo de 1955. La técnica, siguiendo a Haagensen y descrita por sus iniciadores en el Instituto, doctores Rebhein y Castro (13), es como sigue:

Con anestesia general, con intubación endotraqueal (por posible herida en la pleura), se practica la primera biopsia en el seno, por medio de una pequeña incisión practicada directamente sobre el tumor, $\mathrm{y} \cdot$ resecando una pequeña cuña de 5 a 10 milí- 
metros. Se extirpará el tumor completo, cuando éste sea menor cle un centímetro. Se practica hemostasis cuidadosa antes del cierre de la piel.

Es preciso retirar ese instrumental usado, para evitar la reimplantación de células cancerosas en sitios sanos.

Si el resultado histológico es positivo para malignidad, se procede a practicar las otras dos biopsias.

La segunda corresponderá a la cadena de la mamaria interna. Se practica por medio de una incisión paralela al borde del esternón y a 1 centímetro del mismo, que cubre los 3 primeros espacios, desde el borde inferior del primer cartílago al superior del cuarto.

Se incide tejido celular subcutáneo y luego se divulsionan las fibras del pectoral mayor, tomando como reparo el segundo cartílago.

Se exploran el segundo y tercer espacio en su orden, ya que el primero es el más difícil, y el segundo el más constante para la metástasis. Se secciona el músculo intercostal correspondiente, cayendo en la fascia endotorácica, sobre la cual reposan los ganglios y los vasos mamarios internos. Se extirpe el.tejido celuloganglionar y se procede a cerrar la herida sin necesidad de drenaje.

El doctor Muñoz, del departamento quirúrgico (14), ha modificado un poco la técnica, resecando el segundo cartilago costal para abordar al mismo tiempo el segundo y tercer espacios:

Se utiliza la misma incisión. Se incide el pericondrio y con un elevador de periostio se libera el cartílago completamente en sus dos caras. Se secciona el cartilago con bisturí en su inserción esternal y en la articulación condrocostal y se extrae. Se reconocen los vasos mamarios y se extirpan los ganglios.

Esta última técnica es la que más se ha empleado, por su mayor amplitud y facilidad para explorar al mismo tiempo los espacios intercostales, extirpar los ganglios y el menor riesgo zara herir los vasos mamarios y la pleura.

La tercera biopsia, en la fosa supraclavicular, se hace por medio de una incisión de unos 5 centímetros, a 1 centímetro del borde superior de la clavicula y en su parte media. Se secciona tejido celular subcutáneo y aponeurosis cervical. Se rechaza hacia adentro el músculo esternocleidomastoideo y se extirpa el tejido celucloganglionar en el espacio comprendido por las venas yugular y subclavia y el vientre posterior del omohioideo. 
Cuando se quiera practicar la biopsia del vértice de la axila en vez de la supraclavicular, se hace una incisión de 4 a 6 centimetros y 2 centímetros por debajo de la parte media de la clavícula. Se retrae hacia abajo el músculo pectoral mayor. Se incide la fascia axilar y se extirpan los ganglios que se puedan encontrar en el área limitada hacia arriba por la vena axilar: adentro y abajo por la pared costal y hacia afuera por los vasos acromiotorácicos.

En los 43 casos a quienes se practicó la triple biopsia, clínicamente 33 presentaban metástasis axilares.

A continuación presentamos un cuadro sobre los tratamien-. tos practicados y los hallazgos en las metástasis ganglionares.

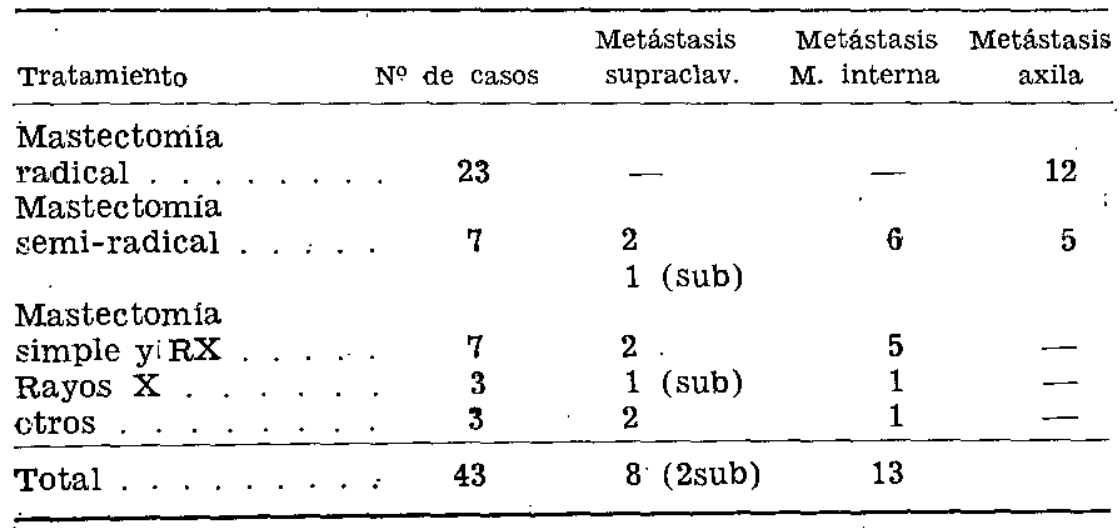

Sobre los 30 casos a quienes se practicó disección de la axila, 17 fueron positivos para metástasis axilares. Se practicaron 3 biopsias subclaviculares, encontrándose metástasis en 2.

En estos 43 casos, considerados clinicamente operables, tan solo y por medio de la triple biopsia se pudo practicar la mastectomia radical en 23 casos, correspondiendo a un porcentaje de $53.4 \%$, es decir, que un $46.6 \%$ estaba destinado a fracasar en caso de que se hubiese practicado la mastectomia radical indiscriminadamente, en esos casos considerados inicialmente como clínicamente operables. 


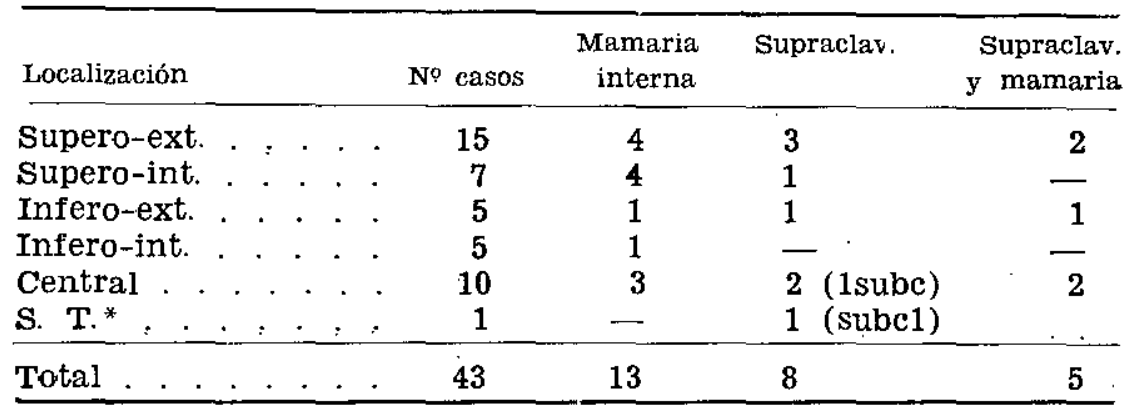

S. T.* Sin tumor por mastectomía previa fuera del Instituto.

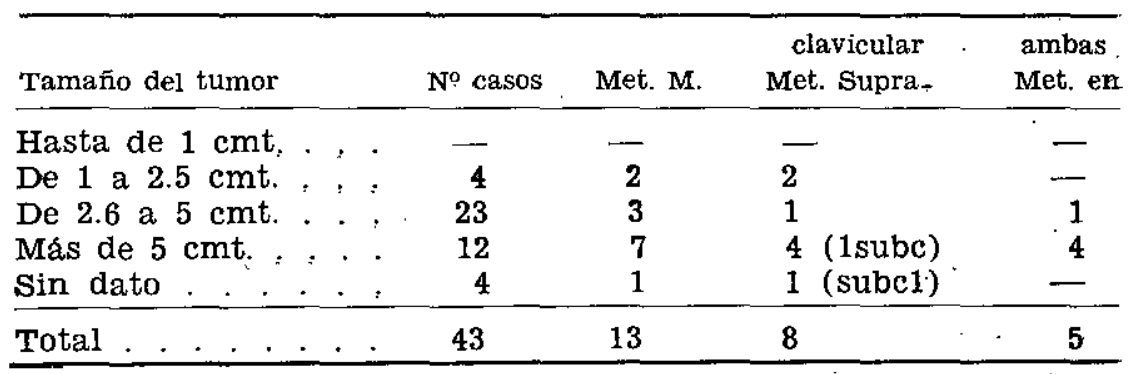

En cuanto a la presencia de metástasis, encontramos su más alto porcentaje en los tumores localizados en el cuadrante supero-interno y en la región central y su mayor frecuencia en relación directa con el aumento de tamaño del tumor.

El procedimiento de la triple biopsia evidentemente inocuo, no produjo ninguna complicación en los casos presentados.

Se practicó como método auxiliar de indiscutible valor para seleccionar los casos considerados quirúrgicos, y practicar entonces la mastectomia radical cuando la biopsia para ganglios de mamaria interna y supraclaviculares fuese negativa.

Además la mastectomía radical, se practicará a los casos cuyo estudio clínico permita practicar el acto quirúrgico y que no presenten los siguientes signos de acuerdo con Haagensen:

1. Edema en la totalidad de la piel del seno.

2. Nódulos satélites en la piel.

3. Carcinoma de tipo inflamatorio.

4. Edema del brazo.

5. Dos o más de los siguientes signos:

a) Ulceración de la piel.

b) Edema de la piel del seno en menos de una tercera parte de su superficie. 
c) Fijación del tumor a la pared costal.

d) Ganglios axilares metastásicos de más de 2.5 centímetros de diámetro.

e) Fijación de los ganglios axilares a la piel o a las estructuras profundas.

6. Metástasis a distancia.

En los casos restantes se practicará la mastectomía simple, seguida de radioterapia y en los estados avanzados los métodos paliativos anotados inicialmente.

\section{BIBLIOGRAFIA}

1. FORERO LAVERDE H.-Cáncer de la glándula mamaria. Tesis de grado. Pontificia Universidad Javeriana. Bogotá, 1953.

2. JACOME VALDERRAMA J. A., MUÑOZ J. J., FORERO LAVERDE H.-Cáncer del seno. Rev. Col. de Cancerología. 1: 112, 1954.

3. PEARSON C. D., WEST, J. P., MC LEAN, M. C. LI and M. B. LIPSETT. Endocrine treatment of metastatio breast cancer. Ann Surgeon, 21: 1.075, 1955.

4. JESSIMAN ANDREW and MOORE FRANCIS-Carcinoma of the breast. New England Journal of Medicine. Medical progress series. 21, 1956.

5. TOBIASSEN, B. SORENSEN and E. HASNER-Acta chirurgica Scandinava. Vol. 3. Fasc. 6, 456, 1956.

6. HAAGENSEN C. D.-Diseases of the breast. W. B. Saunders Company. Fhiladelphia and London, 1956.

7. COLLINS V. P.-Breast Cancer: The influence of Treatment that Fails to cure. Cancer $N^{\circ} 6,9: 1.177,1956$.

8. OWEN H., WAGENSTEEN, F., JOHN LEWIS and STUART w. ARHELGER.-The extended or super radical mastectomy for carcinoma of the breast.

The surgical clinics of North America. 1.051, August, 1956.

9. JEROME A. URBAN.-Radical mastectomy with en bloc in continuity resection of the internal mammary limph node chain. The Surgical Clinics of North America. 1.065. August. 1956.

10. HANDLEY R. S., and THACKRAY A. C.-Invasion of the internal mammary limph glands in carcinoma of the breast. Brit. J. Cancer 1: 15, 1947'

11. R. S. HANDLEY, D. H. PATEY, and B. H. HAND-Excision of the Internal mammary chain in radical mastectomy. The Lancet. $\mathrm{N}^{\circ} 16.1: 457$, 1956.

12. FRANK E. ADAIR -Cancer of the breast. Surgical clinics of North America. 313, 1956.

13. LUIS E. CASTRO y ALFREDO REBHEIN.-Metástasis del carcinoma del seno a los ganglios supraclaviculares y de la cadena mamaria interna. Significado de la triple biopsia. Revista de la Facultad de Medicina, Universidad Nacional, 6 y 7 . Volumen XXIII: 301, 1956.

14. LUIS MARTINEZ CABALLERO-El problema de las metástasis ganglionares regionales en el carcinoma del seno y su tratamiento. 'Tesis de grado. P. U. C. J. Bogotá, 1955. 